\title{
AN INERTIAL FUSION PROPULSION SCHEME FOR SOLAR SYSTEM EXPLORATION
}

\author{
Terry Kammash and David L. Galbraith \\ Department of Nuclear Engineering \\ The University of Michigan \\ Ann Arbor, MI 48109
}

(313) 764-0205

\begin{abstract}
A novel fusion scheme that combines the favorable aspects of both inertial and magnetic confinement approaches is analyzed as a propulsion device for potential utilization in solar system exploration. Using an appropriate set of equations for the plasma dynamics and the magnetic nozzle, we assess the system's propulsive capability by applying the results to a round trip mission to Mars. We find that such a device would allow a massive vehicle to make the journey in less than five months.
\end{abstract}

\section{INTRODUCTION}

It is well known that next to antimatter annihilation reactions, nuclear fusion reactions involving hydrogen isotopes have the highest specific energy $\left(3.5 \times 10^{14} \mathrm{~J} / \mathrm{kg}\right)$ of any fuel considered suitable for propulsion purposes. Although no power producing fusion reactors have yet been built, it is believed on the basis of the research progress that has been made thus far, that such a system will become a reality in the early part of the next century. Of the two major approaches that are being pursued world wide, namely magnetic and inertial confinement fusions, the latter appears to be more suitable for propulsion because of its unique capability and relative simplicity (Kammash and Galbraith 1988). The basic requirement of such a system, whether as a terrestrial power source or as a propulsion device, is that it generates significantly more energy than it consumes, that is, it possesses a large gain factor " $Q$ ". In the case of propulsion large $Q$ value means high fuel (plasma) temperature which in turn means very large specific impulse, $I_{s p}$, a characteristic deemed highly desirable for space exploration.

One of the most novel and promising fusion schemes that have surfaced recently is the Magnetically Insulated Inertial Confinement Fusion (MICF) concept illustrated in Figure 1. In this scheme a hot plasma is generated inside a target pellet, whose walls are coated with fusion fuel, as a result of ablation initiated by a laser beam that enters the target through a hole. Not only does this laser beam create the plasma, but it also gives rise to a strong, instantaneous magnetic field that serves to thermally insulate the hot plasma from the metal shell that contains it. The lifetime of such a plasma is dictated by the shock speed in the shell rather than by the sound speed in the plasma itself as is the case in the standard implosion-type inertial fusion. This translates to about two orders of magnitude longer confinement time due to the larger atomic mass of the metal shell, on the one hand, and a lower shell temperature arising from the magnetic field insulation on the other. As a result the plasma is allowed to burn much longer, producing more fusion energy and correspondingly large gain factors (Kammash and Galbraith 1989). The MICF scheme has been experimentally studied at Osaka University in Japan where high temperature plasma along with an insulating magnetic field have been generated, and sizable neutron production from deuterium-deuterium (DD) reactions have been observed (Nishihara et al. 1988). The fusion parameters, namely plasma density, temperature, and confinement time, that characterize an energy breakeven $(Q=1)$ are within reach of the Osaka experiment, and there is every reason to 


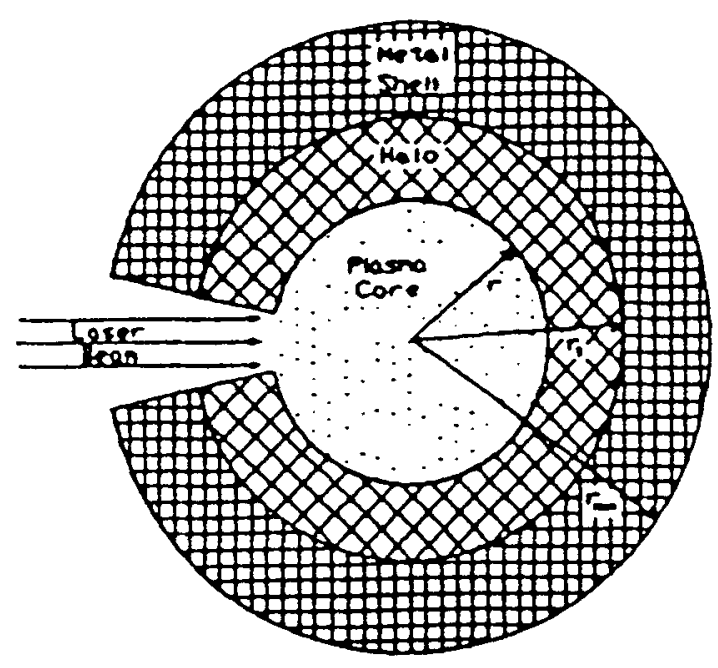

(a)

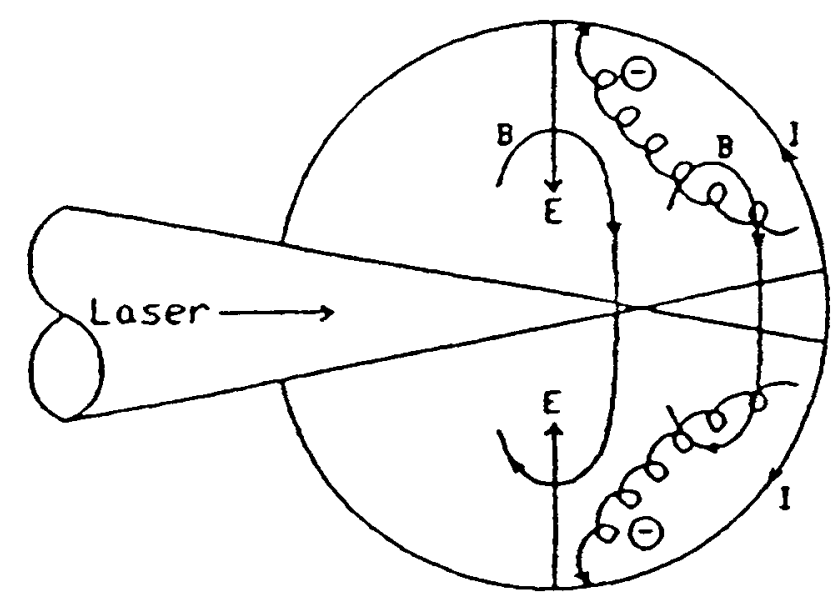

(b)

FIGURE 1. Schematic Diagram of (a) Plasma Formation and (b) Magnetic Field Formation in MICF. 
believe that such a condition will be achieved in the not too distant future. As noted earlier, $Q \gg>1$ is needed for a propulsion device, and if progress is sustained at its present level, it can be safely said that MICF will eventually produce those Q-values needed for the propulsion parameters required to explore the solar system.

In this paper we utilize a physics model we developed earlier (Kammash and Galbraith 1989) to assess the propulsive capability of MICF. This model consists of a set of quasi onedimensional time dependent conservation equations that describe the dynamics of the fusion plasma in a multi-region pellet that, when solved, produces the plasma properties at the end of the burn as well as the Q-value. We use these fusion parameters to calculate the propulsion parameters of MICF which we ultimately test by applying them to a round trip mission to Mars.

\section{ROCKET PROPULSION BY MICE}

A schematic diagram depicting a rocket driven by an MICF propulsion device is shown in Figure 2. The main elements in this system are the pellet injector, the laser, the reaction chamber and the magnetic nozzle. The principle of operation of such a rocket is that pellets containing fusion fuel such as deuterium-tritium (DT) are injected at a predetermined rate $(\omega)$ into the reaction chamber where they are zapped by a laser beam of specific input energy $\left(E_{i n}\right)$ and other properties guaranteed to initiate the fusion burn in the target. At the end of the burn the hot plasma consisting of the fuel ions, electrons, and the metal shell ions is allowed to adiabatically expand in the reaction chamber where a carefully programmed, externally applied magnetic field that constitutes the magnetic nozzle traps these charged particles and guides them to the throat where they are ejected. We choose an azimuthally symmetric magnetic configuration and deduce the basic propulsion parameters using an axisymmetric magnetohydrodynamic (MHD) flow model for a plasma with very small electrical resistivity. At the high temperatures achieved at the end of the fusion burn, the plasma will indeed be highly conducting allowing it to move along the field lines of the nozzle, and by specifying the dimension of the throat and the reaction chamber we can calculate the time it takes the individual species to escape through the nozzle throat. It can be readily shown that most of the DT fusion energy generated in MICF appears in the neutrons (which are not constrained by the magnetic field) and the electrons which escape through the nozzle at very high speeds but contribute very little to the thrust generated by the system due to their small mass. On the other hand the fuel ions, and predominantly the shell ions, are the ones that provide the major portion of the thrust but at the expense of small exhaust velocities which translate to a smaller specific impulse than would have been the case with electrons only. In view of this, the time it takes the heaviest ions to escape dictates the maximum rep rate at which the system must operate, and along with the fuel ions they dictate the thrust provided to the rocket by the MICF driver. For the purposes of this paper we assume that the neutron energy is disposed of through radiators, but it is clear that if converted (typically at about $40 \%$ efficiency) through a thermal conversion scheme to electric power, the neutron portion of the fusion energy constitutes a major source of electric power that can be carried on board.

It is often argued that the neutrons produced by the fusion reactions are undesirable since they can induce radioactivity that is hazardous to the crew, and can also interfere with the functioning of the electronic and navigational equipment. To minimize this effect it might be necessary to consider alternative fusion fuel cycles such as the catalyzed DD or deuterium-helium $3\left(\mathrm{DHe}^{3}\right.$ ) where neutron production can be reduced to a few percent of the fusion energy compared to the $80 \%$ in the case of DT. The potential use of the $\mathrm{DHe}^{3}$ as a fuel for an MICF propulsion device has been examined in a preliminary fashion (Kammash and Galbraith 1990) where it was shown that due to the high temperature required for ignition, and correspondingly the large laser input energy needed, can significantly increase the dry weight of the vehicle and in turn the mission duration. It has been argued (Hyde 1983) that at such high temperatures there is copious production of energetic $\mathrm{X}$-rays due to bremsstrahlung which also require extensive shielding thereby negating the substantial advantage often cited for the $\mathrm{DHe}^{3}$ fuel cycle. Although these 


\section{MICF FUSION PROPULSION SYSTEM}
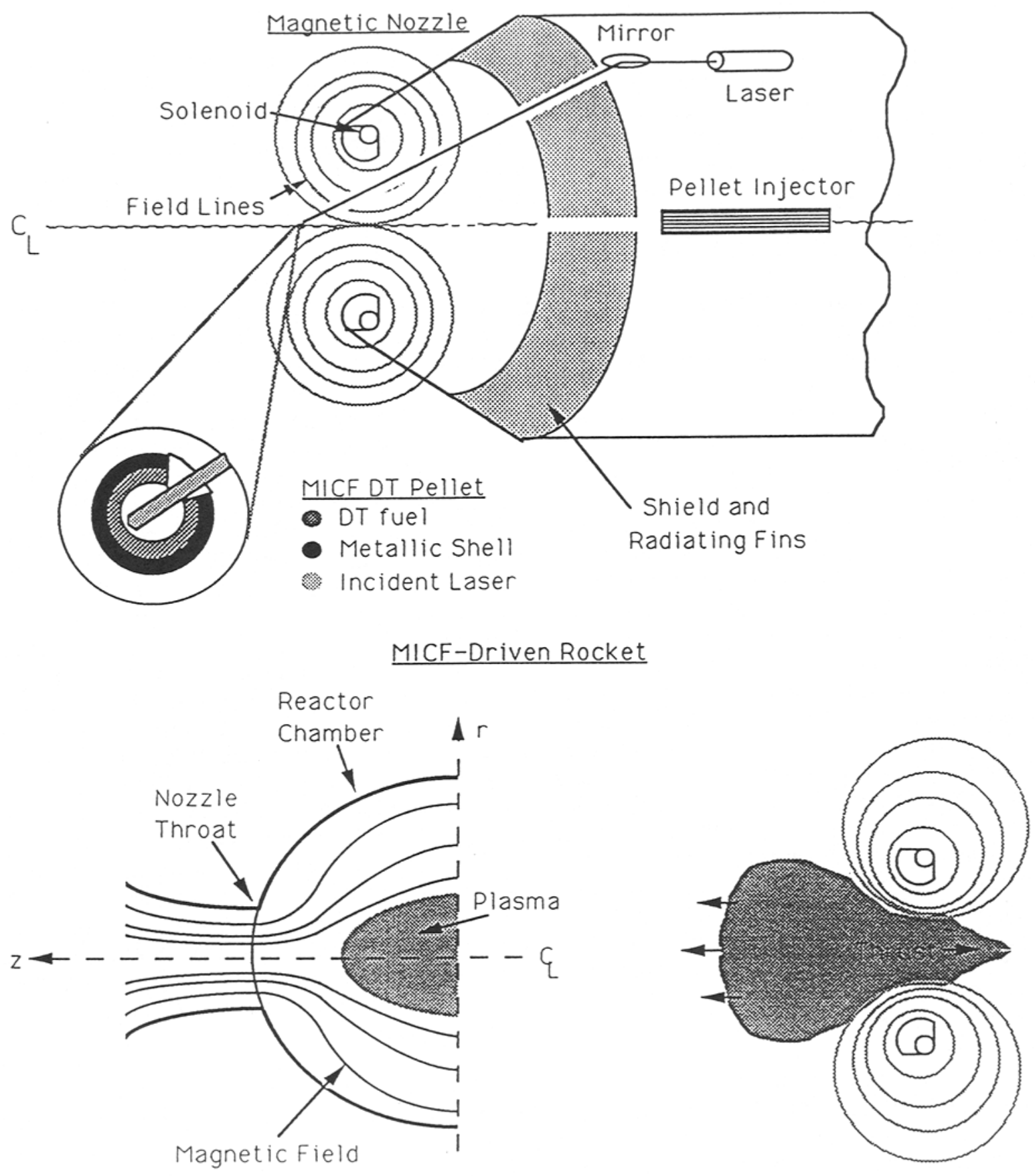

Meridional Magnetic Nozzle

FIGURE 2. Schematic of MICF Rocket and Magnetic Nozzle. 
arguments appear on the surface to be plausible, only a detailed study with appropriate pellet designs can truly establish the merit of each fuel cycle especially as it pertains to propulsion. We have seen, for example, that in MICF the final plasma temperature can be significantly lower than the initial ignition temperature due to the fact that the metal shell remains quite cold throughout the burn. This means that the temperature achieved by the system due to thermalization of all the species at the end of the burn can be sufficiently low not to cause any major concern with radiation emission. We have in the current study also explored the potential utilization of catalyzed DD in an MICF propulsion system and the results will be compared to those of DT in our discussion of the applications.

\section{APPLICATION TO MANNED MARS MISSION}

The potential utilization of MICF for advanced space propulsion can be assessed by evaluating its performance as a driver for a manned mission to the planet Mars. Its propulsive capability depends critically on its performance as an energy producer, and for that we make use of the physics model, alluded to earlier, to generate the fusion plasma parameters for DT and catalyzed $\mathrm{DD}$ fuel cycle for the pellet design given in Table 1.

TABLE 1. Pellet and Plasma Parameters.

\begin{tabular}{lll}
\hline & DT & Cat DD \\
\hline & & \\
Inner radius of fusion fuel & $0.25 \mathrm{~cm}$ & $0.25 \mathrm{~cm}$ \\
Outer radius of fusion fuel & $0.30 \mathrm{~cm}$ & $0.30 \mathrm{~cm}$ \\
Outer radius of metal shell & $0.547 \mathrm{~cm}$ & $0.495 \mathrm{~cm}$ \\
Input laser energy & $2.59 \mathrm{MJ}$ & $4.56 \mathrm{MJ}$ \\
Initial plasma density & $5 \times 10^{21} \mathrm{~cm}^{-3}$ & $3 \times 10^{21} \mathrm{~cm}^{-3}$ \\
Initial plasma temperature & $11.785 \mathrm{keV}$ & $40 \mathrm{keV}$ \\
Total pellet mass & $8.75 \mathrm{~g}$ & $6.03 \mathrm{~g}$ \\
Energy gain factor & 724 & 82 \\
Average ion energy at end of burn & $1.10 \mathrm{keV}$ & $0.735 \mathrm{keV}$ \\
Reaction chamber volume & $4.189 \times 10^{3} \mathrm{~cm}^{3}$ & $4.189 \times 10^{3} \mathrm{~cm}^{3}$ \\
Nozzle throat area & $19.63 \mathrm{~cm}^{2}$ & $19.63 \mathrm{~cm}^{2}$ \\
& & \\
\hline
\end{tabular}

As noted earlier, the plasma at the end of the burn will contain the fusion fuel ions, the electrons as well as the ions resulting from the total ionization of the metal shell (tungsten). These metal ions will have on the average the same energy as the plasma particles due to full thermalization of the species that takes place at the high temperature generated by the fusion reactions. Because of their small mass the electrons contribute little to the thrust although they assume a major portion of the fusion energy. Moreover, because of their large mass the metal ions acquire the smaller thermal velocities and in turn dictate the escape time from the reaction chamber as well as the thrust of the device. Making use of ideal MHD and the properties of an azimuthally symmetric magnetic nozzle whose throat area is given in Table 1, we calculate the thrust as a function of the pressure at the reservoir (center of reaction chamber) which is the total plasma pressure achieved at the end of the burn. These propulsion parameters are given in Table 2. 
TABLE 2. MICF Propulsion Parameters.

DT

Cat DD

Fuel ion exhaust velocity

Tungsten ion exhaust velocity

Effective specific impulse, Isp

Firing rate, $\omega$

Total thrust, $\mathrm{F}$

Jet power, $\mathrm{P}_{\text {jet }}$
$376 \mathrm{~km} / \mathrm{s}$

$43.8 \mathrm{~km} / \mathrm{s}$

$0.451 \times 10^{4} \mathrm{~s}$

$\leq 6422 \mathrm{~s}^{-1}$

$0.412 \times 10^{3} \omega \mathrm{N}$

$9.70 \omega \mathrm{M} \mathrm{W}$
$344 \mathrm{~km} / \mathrm{s}$

$35.9 \mathrm{~km} / \mathrm{s}$

$0.370 \times 10^{4} \mathrm{~s}$

$\leq 5254 \mathrm{~s}^{-1}$

$0.233 \times 10^{3} \omega \mathrm{N}$

$4.627 \omega \mathrm{M} \mathrm{W}$

We apply these results to a round trip mission from earth to Mars using $\omega=100$. Since MICF is laser driven the dry weight of the vehicle must include the power supply system, and at an input energy of $2.6 \mathrm{MJ}$ such a system including the driver (lasers, radiators, optics, energy handling), the thrust chamber, and the overhead components are estimated (Hyde 1983) to be 564 megagrams $(\mathrm{Mg})$. We arbitrarily add $100 \mathrm{Ms}$ payload and assume that the dry weight for the mission vehicle is $664 \mathrm{Mg}$. We also consider a continuous burn acceleration/deceleration trajectory profile which assumes constant $\mathrm{I}_{\mathrm{sp}}, \mathrm{F}$, and $\mathrm{P}_{\text {jet }}$ operation. The equations that describe the transit time for the outbound and return legs of the journey can be readily derived (Borowski $1987)$, and knowing the linear distance from Earth to Mars $\left(0.52 \mathrm{AU}=7.8 \times 10^{10} \mathrm{~m}\right)$ we find that the round trip journey for this MICF-driven vehicle takes 138 days in the case of DT, and 171 days for Cat DD. In obtaining the second result we used the same dry weight on the assumption that a reduction in the neutron energy from $80 \%$ to $54 \%$ reflected in less shielding and radiators offsets the increase in weight demanded by the larger laser input energy. No attempt was made to optimize the performance of the driver, and correspondingly the travel time. This can be achieved by a careful pellet design since an optimum balance between the specific impulse and the thrust can be achieved by proper choice of the dimensions of the fuel and the metal shell (as well as the choice of the metal component itself) consistent with maximum production of fusion energy at minimum laser input energy.

\section{CONCLUSION}

With the aid of an extensive fusion physics model and an appropriate magnetic nozzle design, the propulsion capability of the Magnetically Insulated Inertial Confinement Fusion Concept was analyzed and evaluated for potential utilization in the space explorations contemplated for the early part of the next century. It is shown that such a device can produce highly desirable propulsion characteristics that would allow a sizable vehicle to make a round trip to Mars in less than five months. It is also shown that catalyzed DD fuel results in a somewhat less propulsion performance than DT though at a significantly lower neutron production. The velocity increment, $\Delta \mathrm{V}$, generated by this system and the corresponding trip time are in excellent agreement with the predictions of Irving and Blum (1959). Reduction in journey time can be achieved in both instances, however, with appropriate and feasible optimization of the system. 


\section{Acknowledgments}

This work was supported in part by the National Aeronautics and Space Administration.

\section{References}

Borowski, S. (1987) "A Comparison of Fusion/Anti Proton Propulsion Systems for Interplanetary Travel," AIAA paper 87-1814, AIAA/SAE/ASME/ASEE 23rd Joint Propulsion Conference, held in San Diego, CA, 29 June-2 July, 1987.

Hyde, R. A. (1983) A Laser Fusion Rocket for Interplanetary Propulsion, Report UCRL-88857, Lawrence Livermore National Laboratory, Livermore, CA.

Irving, J. H. and E. K. Blum (1959) "Comparative Performance of Ballistic and Low Thrust Vehicles for Flights to Mars," Vistas in Astronautics, Vol. II, New York: Pergamon Press, p. 191.

Kammash, T. and D. L. Galbraith (1988) "A Promising Fusion Approach to Advanced Space Propulsion," L. British Interplanetary Society, 41: 327-533.

Kammash, T. and D. L. Galbraith (1989) "A High Gain Fusion Reactor Based on the Magnetically Insulated Inertial Confinement Fusion Concept," Nuclear Fusion, 29: 1079-1099.

Kammash, T. and D. L. Galbraith (1990) "Advanced Fuels for An Inertial Fusion Propulsion System," in Trans. 7th Symposium on Space Nuclear Power Systems, CONF-900109, held in Albuquerque, NM, 7-10 January 1990, pp. 61-65.

Nishihara, K. H. Daido et al. (1988) "Magnetically Insulated Inertially Confined Fusion by Long Wave Length Lasers and Multiregion Analysis of MICF Reactor," in Proc. 12th International Conference on Plasma Physics and Controlled Nuclear Fusion, held in Nice, France, 12-19 October 1988, Vol. 3, p. 89. 